\title{
Candida Pneumonia with Lung Abscess as a Complication of Severe COVID-19 Pneumonia
}

\author{
Adel Abdelhadi $\mathbb{D}^{\prime}$ \\ Abeer Kassem ${ }^{2}$ \\ 'Department of Critical Care Medicine, \\ Saqr Hospital, MOH, Ras Al-Khaimah, \\ Ras Al-Khaimah, United Arab Emirates; \\ ${ }^{2}$ Department of Pulmonary Medicine, \\ IBHOA Hospital, MOH, Ras Al-Khaimah, \\ Ras Al-Khaimah, United Arab Emirates
}

\begin{abstract}
A South Asian male patient in his mid-forties presented with symptoms of severe 2019-nCoV (COVID-19) and recent brain infarction. Subsequently, he was found to have evidence of sepsis, underlying undetected diabetes mellitus (DM) and oral candida mucositis, possibly leading to the rare occurrence of direct spread to the lung, manifesting as a necrotizing candida lung abscess. We describe the diagnosis, clinical course, and management of the unique complication in this case that occurred during his admission, hospitalization, and eventual successful discharge from the hospital. This case highlights the importance of early identification and treatment of suspected COVID-19 infection based on clinical and radiological assessments before the confirmation of COVID-19 by real-time polymerase chain reaction (rtPCR) test result, especially in patients with hyperglycemia. It also indicates the complications that can occur due to COVID-19 such as arteriovenous manifestations and the rare occurrence of pulmonary candida lung abscess. Early detection and prompt management by interdisciplinary teams in the emergency room, followed by close monitoring of complications in the intensive care unit (ICU), can lead to successful outcomes in severe/ critical COVID-19 infection.
\end{abstract}

Keywords: Candida albicans, management, computed tomography scan, remdesivir therapy

\section{Introduction}

An outbreak of a cluster of cases of pneumonia from an unknown cause in Wuhan City, Hubei Province of China, was reported to the World Health Organization (WHO) China office on December 31, 2019. ${ }^{1}$ Most of the affected patients were associated with the local Huanan Seafood market with the main clinical signs and symptoms of fever, with a few patients having difficulty in breathing. Invasive lesions were seen in the lungs of few patients on chest radiographs. ${ }^{1}$ On January 7 , 2020, Chinese health authorities were able to isolate and confirm that patients in this cluster suffered from a novel coronavirus, 2019-nCoV, after having ruled out other known respiratory pathogens. ${ }^{2}$ The virus then spread rapidly around the globe, leading the WHO Director-General to declare the virus as a pandemic on March 11, 2020. ${ }^{3}$ Fever, cough, chest pain, shortness of breath represent the common symptoms of COVID-19. ${ }^{4}$ While majority of the patients affected by this virus have mild symptoms with good prognosis, around $10-15 \%$ of the cases worldwide develop severe manifestations, such as acute respiratory syndrome (ARDS), pneumonia, multiple organ failure, coagulation, and septic shock that might lead to high mortality rates. ${ }^{4-6}$ Moreover, severity of the disease and the mortality rates are found to be higher in COVID-19 patients with coexisting comorbidities like DM, asthma, obesity, etc. Critically-ill COVID-19 patients
Department of Critical Care Medicine, Saqr Hospital, MOH, Ras Al-Khaimah, Ras Al-Khaimah, United Arab Emirates Tel +97172049868

Fax +97142574933

Email adeltaha72@gmail.com 
with ARDS are also reported to be more susceptible to fungal super-infections caused by Candida species and Aspergillus fumigatus. ${ }^{7,8}$ Invasion of internal organs by Candida species might occur in patients with severe COVID-19 due to excessive use of antibiotics and corticosteroids, and central venous catheter, coupled with the complications caused by severe ARDS coronavirus 2 (SARS CoV-2). ${ }^{9}$ Besides candidiasis and aspergillosis, occurrence of mucormycosis (also known as "black fungus") has been reported recently in Asian countries (mostly in India) among patients affected with COVID19. ${ }^{10}$ The most common risk factor associated with mucormycosis is DM. The use of glucocorticoids to treat COVID-19 in patients with DM has been reported to be linked with the development of mucormycosis ${ }^{11}$ In this case report, we present the treatment course of a patient with severe COVID-19 suffering from progressive shortness of breath, mouth ulcer, scrotal swelling followed by respiratory distress and Candida infection and successful management by an integrated multispecialty team.

\section{Case Presentation, Management, and Follow-Up}

A 40-year-old South Asian male patient, with no previous history of any chronic illness was presented to the emergency department of our hospital with sudden altered consciousness, high fever, and progressive shortness of breath for over 5 days on 14.07.2020. He was apparently healthy until admission and was not taking any regular medications and had no history of drug allergy. The patient was febrile with a temperature of $39.6^{\circ} \mathrm{C}$, heart rate of 140 beats/minute, and oxygen saturation $\left(\mathrm{SpO}_{2}\right)$ of $72 \%$ in room air. The patient appeared thin and cachectic, weighed $62 \mathrm{~kg}$, indicating poor nutritional status. Notable observations included numerous mouth ulcers and scrotal swelling with redness and tenderness. The mouth ulcers were clinically diagnosed as Candida mucositis by the dermatologist. The patient was considered for emergency admission and the test for COVID-19, real-time polymerase chain reaction (rtPCR) and chest Computed Tomography (CT) were done as per our hospital admission protocol. The patient was also screened for HIV upon admission as per routine protocol and tested negative. Since the patient tested negative for HIV, the patient was not tested for T-lymphocyte subsets. A timeline of key events for this case is depicted in Figure 1.
The patient was subjected to an ultrasonogram (USG) that confirmed scrotal edema, but all other parameters in the USG were reported as normal. There was, however, remarkable elevation of inflammatory markers indicative of infection. A brain CT showed a recent right basal ganglia infarct (Figure 2), which seemed to be 48 to 72 hours old. The chest CT was highly suggestive of COVID-19 with COVID-19 Reporting and Data System (CO-RADS) score of 5 (Figure 3). Laboratory investigations revealed total leukocyte count (TLC) of $25 \times 10^{9} / \mathrm{L}$ (normal: $4.5-$ $10 \times 10^{9} / \mathrm{L}$ ), hemoglobin $5.34 \mathrm{mmol} / \mathrm{L}$ (normal: $8.7-11.2$ $\mathrm{mmol} / \mathrm{L}$ ) and platelets $128 \times 10^{9} / \mathrm{L}$ (normal: $150-400$ $\left.\times 10^{9} / \mathrm{L}\right)$. C-reactive protein (CRP) and procalcitonin levels were $1750 \mathrm{mg} / \mathrm{L}$ (normal: $8-30 \mathrm{mg} / \mathrm{L}$ ) and $11 \mathrm{mcg} / \mathrm{L}$ (normal: $\leq 0.15 \mathrm{mcg} / \mathrm{L}$ ), respectively, lipid profile was normal, but the International normalized ratio (INR) was 1.6 (normal: 0.8-1.1 INR). Other elevated values included glycated hemoglobin (HbA1c) 11.3\% (normal:<6.0\%), serum creatinine $170 \mu \mathrm{mol} / \mathrm{L}$ (normal: 53-106 $\mu \mathrm{mol} / \mathrm{L}$ ), serum potassium $6.2 \mathrm{mmol} / \mathrm{L}$ (normal: $3.5-5.5 \mathrm{mmol} / \mathrm{L}$ ), and serum lactate $8 \mathrm{mmol} / \mathrm{L}$ (normal: $0.5-2.2 \mathrm{mmol} / \mathrm{L}$ ). Blood sugar level was $24 \mathrm{mmol} / \mathrm{L}$ (normal: $<7.8 \mathrm{mmol} / \mathrm{L}$ ). Arterial blood gas analysis was suggestive of severe metabolic acidosis [pH: 7.21, partial pressure of carbon dioxide (PaCO2):21 mmHg (normal: 35-45 mmHg), partial pressure of oxygen $\left(\mathrm{PaO}_{2}\right) 58 \mathrm{mmHg}$ (normal: $75-100 \mathrm{mmHg}$ ) and $\mathrm{HCO}_{3}$ was $8 \mathrm{mmol} / \mathrm{L}$ (normal: $23-28 \mathrm{mmol} / \mathrm{L}$ )]. The patient was provisionally diagnosed with sepsis, multiple organ dysfunction, brain infarction, severe viral pneumonia with respiratory failure, uncontrolled DM, oral candidiasis, acute kidney injury, hyperkalemia, severe metabolic acidosis, and hyperlactatemia. Hence, the patient was admitted to the intensive care unit (ICU) on the same day with a Glasgow Coma Scale (GCS) score of $13 / 15$.

In the ICU, the patient was maintained on high flow nasal cannula (HFNC) therapy with a flow rate of $60 \mathrm{~L} / \mathrm{min}$ and a fraction of inspired oxygen $\left(\mathrm{FiO}_{2}\right)$ of $60 \%$ to maintain adequate oxygenation. Anticoagulation was initiated with a high dose of subcutaneous (S.C.) enoxaparin $40 \mathrm{mg}$ BID (bis in die). The patient was also started on intravenous (I. V.) remdesivir $200 \mathrm{mg}$ loading dose followed by $100 \mathrm{mg} \mathrm{I}$. V. daily for 10 days due to high clinical suspicion for COVID-19 infection. Simultaneously, I.V. administration of broad-spectrum antibiotics (tigecycline $100 \mathrm{mg}$ BID, levofloxacin $750 \mathrm{mg}$ BID) for suspicion of skin and soft tissue infection of the scrotum and I.V. anidulafungin $100 \mathrm{mg}$ daily for Candida mucositis was also started, 


\section{Treatment course and Management}

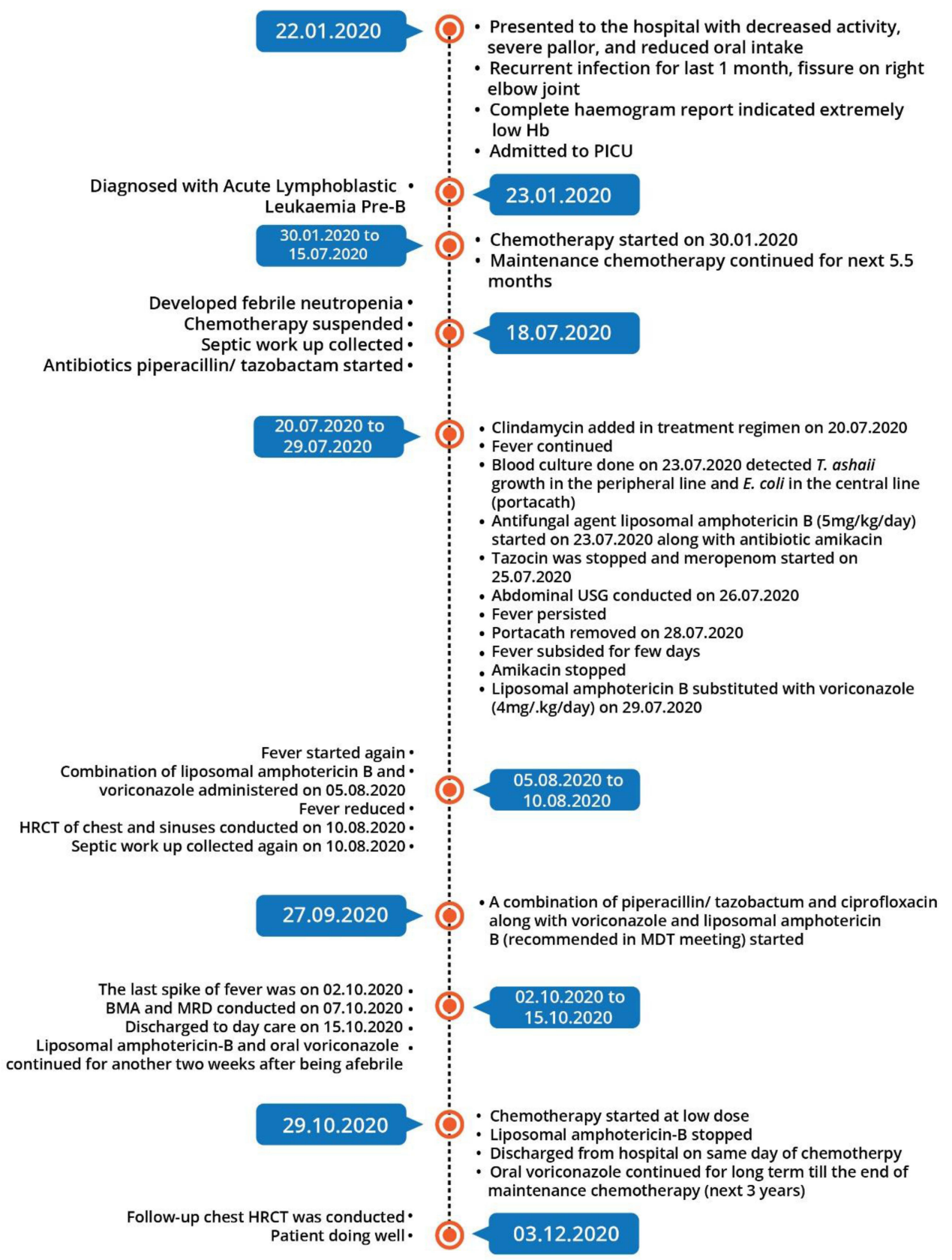

Figure I Key events of the case.

which were to be continued for one week. Diabetes management was done by insulin sliding scale together with insulin glargine. Electrolytes were replenished in the form of magnesium and potassium.

The next day (15.07. 2020), as expected, the COVID19 real-time rtPCR test came back as positive. Left leg swelling was noticed, and the US Doppler study revealed iliofemoral deep vein thrombosis (DVT). Enoxaparin was increased from a high prophylactic dose to a therapeutic dose of S.C. $60 \mathrm{mg}$ BID. Over the next 6 days, the patient regained consciousness and showed remarkable improvement in hemodynamic and ventilatory status. Fever gradually subsided, and hyperglycemia was well-controlled. TLC dropped to $14 \times 10^{9} / \mathrm{L}$, oxygenation improved, and 


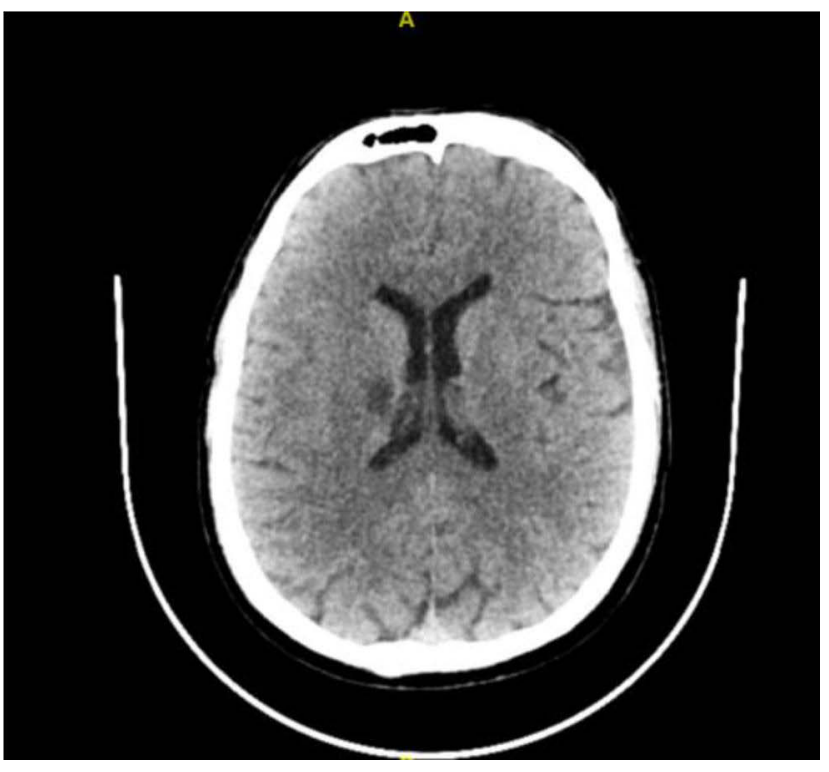

Figure 2 CT of the brain showing evidence of right basal ganglia infarct on 14.07.2020 (day of admission).

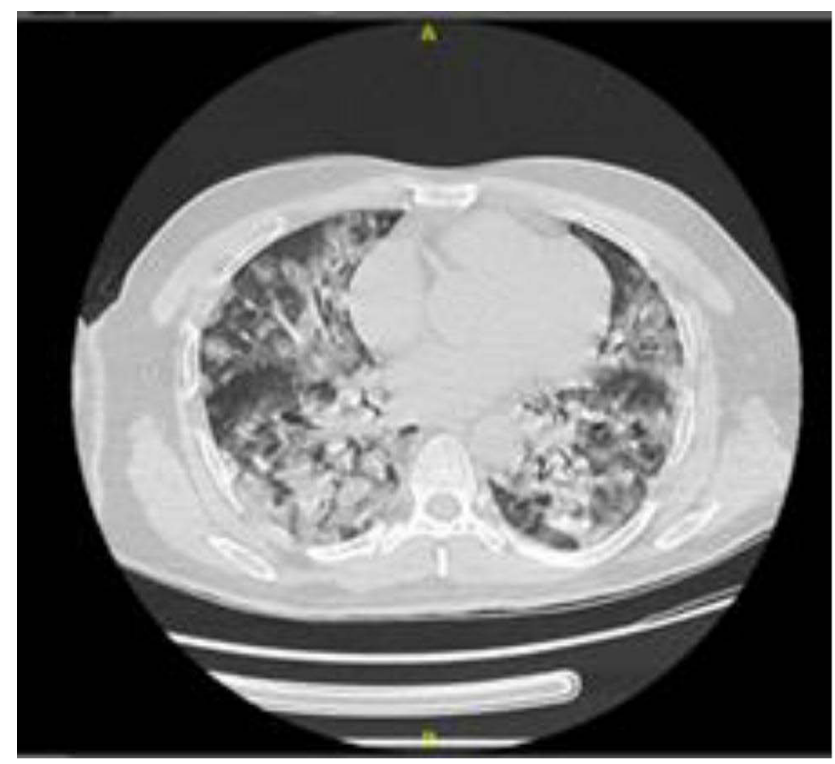

Figure 3 HRCT of chest showing bilateral GGO on 14.07.2020 [CO-RADS (COVID-19 Reporting and Data System) 5].

he was weaned from HFNC to $6 \mathrm{~L} / \mathrm{min}$ nasal cannula. Inflammatory markers like CRP and procalcitonin levels remained high, but gradually improved after adequate sepsis control. Hence, we did not consider anti-cytokine therapy at this point. During this period, he was alternating between supine and self-prone positions.

However, on 21.07.2020 (7 days after admission), clinical deterioration was observed. GCS dropped to 5/15; oxygen requirement increased with signs of hypotension requiring norepinephrine infusion up to $0.4 \mathrm{mcg} / \mathrm{kg} / \mathrm{min}$ for 2 days. TLC increased to $23 \times 10^{9} \mathrm{~L}$. He also suffered from refractory hypoglycemia, which might have occurred due to sepsis, and required frequent administration of dextrose $50 \%$ infusion. Remdesivir therapy was discontinued on 24.07.2020 after a course of 10 days. Chest X-ray showed worsening bilateral infiltrates, more visible in the right lung. Sputum culture was done on 24.07.2020, which showed Klebsiella pneumoniae and Candida albicans. He was diagnosed with hospital-acquired pneumonia (HAP) necessitating invasive mechanical ventilation. Antibiotics were changed to I.V. meropenem 1g TID (ter in die), I. V. linezolid $600 \mathrm{mg}$ BID. Anidulafungin therapy was continued as earlier. He gradually improved over the next few days. Fever subsided, TLC counts dropped to $15 \times 10^{9}$ $\mathrm{L}$, euglycemia was achieved, vasopressors were weaned off, oxygen requirements decreased to $40 \%$ and he was extubated on 26.07.2020. HFNC was continued and the patient was closely monitored with a suggested extended stay in ICU for another week.

On 28.07.2020 (14 days after admission), the patient's oxygenation deteriorated again, and he had to be reintubated. His oxygen requirement was increased from 40 to $70 \%$ to maintain $\mathrm{SpO}_{2}>94 \%$. Due to respiratory deterioration, he was clinically suspected with pulmonary embolism (PE), and a CT angiogram (Figure 4) was requested; however, no evidence of PE was found. A high resolution CT (HRCT) of the chest revealed large cavitary lesions in

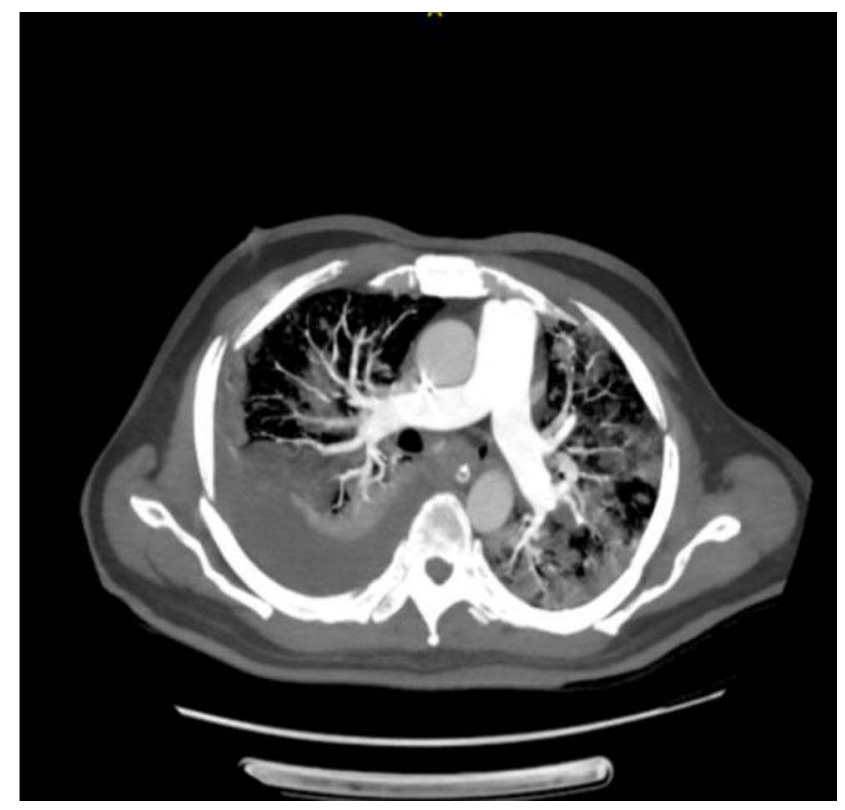

Figure $4 \mathrm{CT}$ angiogram (28.07.2020) showing no evidence of PE. 


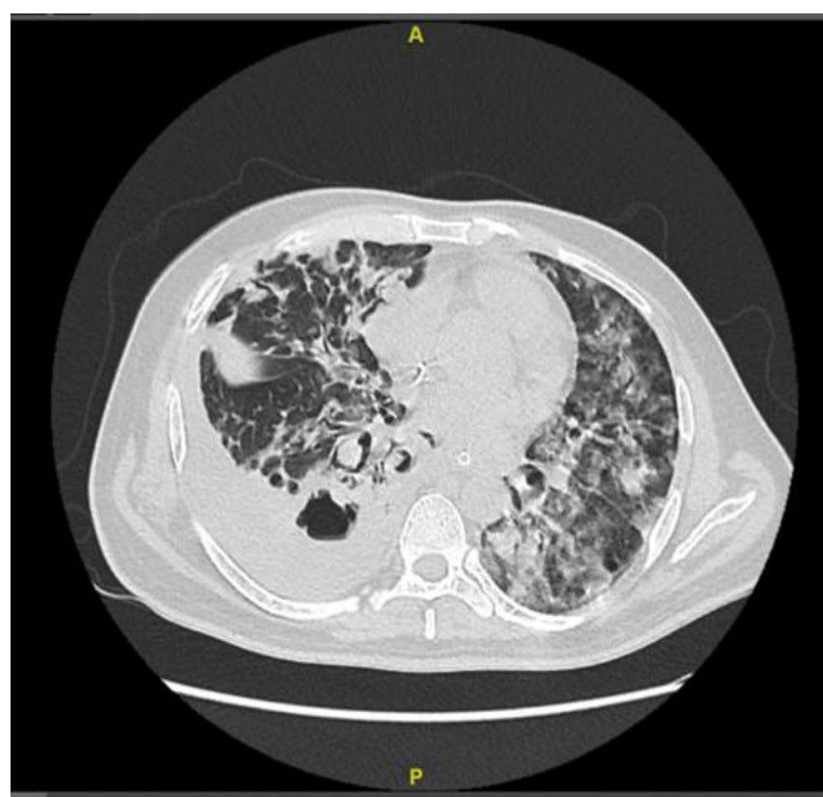

Figure 5 HRCT of chest (28.07.2020) showing right-sided lower lobe lung abscess with right-sided pleural effusion.

the right lung base with pleural effusion denoting necrotizing pneumonia with lung abscess (Figure 5). Right-sided pleural drainage was done by intercostal catheter. Pleural fluid exudate showed no bacterial growth. Fiberoptic bronchoscopy with bronchoalveolar lavage (BAL) was performed, which showed the growth of Candida albicans. The same regimen was continued for anidulafungin and no change was made in the dose of I.V. antibiotics to complete six weeks of antibiotic therapy. The patient once again showed clinical signs of gradual improvement by 03.08.2020 and was extubated on 06.08.2020. We decided to continue I.V. meropenem 1g BID. I.V. linezolid $600 \mathrm{mg}$ BID was replaced with an oral dose of tablet linezolid $600 \mathrm{mg}$ BID, and I.V. anidulafungin was changed to oral voriconazole $200 \mathrm{mg}$ BID for another 6 weeks. He was weaned off HFNC on 09.08.2020 and oxygen support on 18.08.2020 and remained in the ICU until he could be maintained on room air. Throughout his ICU stay, aggressive nutritional support, meticulous titration of anticoagulation doses, and adequate control of DM were also implemented. He was shifted to the regular ward in good shape on 21.08.2020 with a chest X-ray showing remarkable improvement in the resolution of lung lesions (Figure 6). He was discharged from the hospital on 14.09.2020.

\section{Discussion}

During the first two months of the initial outbreak in China, a group of researchers representing the China

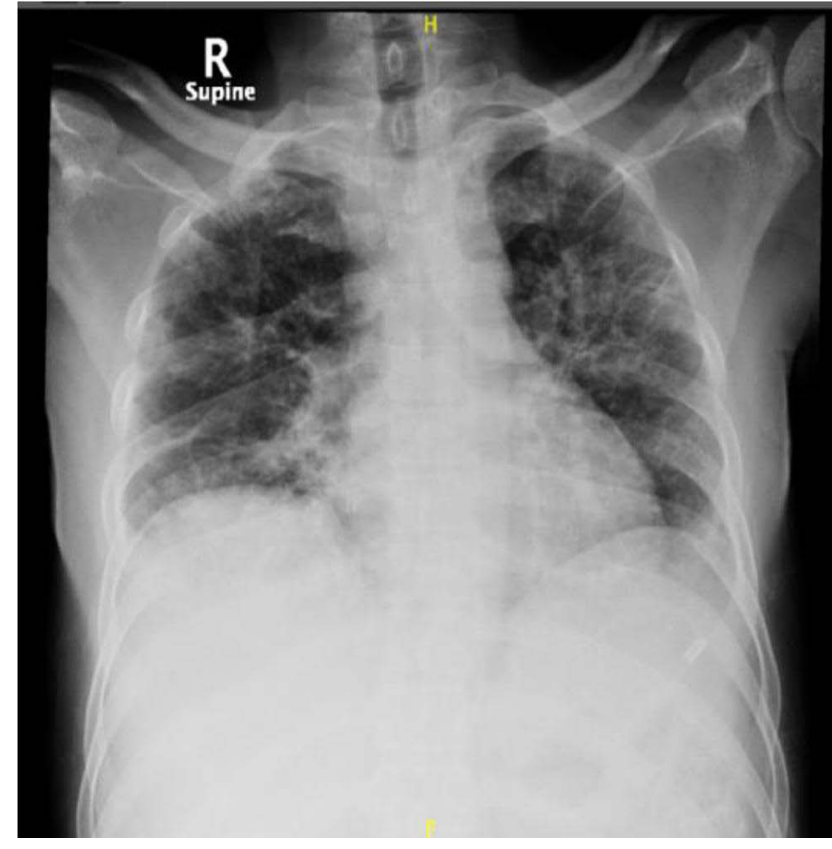

Figure 6 X-ray of chest showing remarkable improvement in the resolution of lung lesions.

Medical Treatment Expert Group for COVID-19 reported varying degrees of severity in patients with COVID-19. ${ }^{12}$ According to a study, majority of the patients with COVID-19 had mild disease with fever and cough being the most common symptoms. In contrast, the patients with severe disease who had a coexisting illness showed high CRP level, which was correlated with disease progression in COVID-19 pneumonia. ${ }^{13}$ CRP levels have been found to increase significantly in patients with severe COVID19 , as the disease progresses. Therefore, concentration of CRP is considered as an important marker of systemic inflammation associated with the severity of disease. ${ }^{14,15}$ If the patient progresses to respiratory failure requiring mechanical ventilation, has clinical signs and symptoms of shock or organ failure, the case is deemed as critical with need of ICU admission for care and management. ${ }^{16}$

Our patient warranted ICU admission due to severity of illness. Chest CT was highly suggestive of COVID-19 infection. Typically, chest CT depicting ground glass opacity (GGO) in the lungs, vascular enlargement, and septal thickening are common indicators of COVID-19 in patients. In rare cases, chest CT of a COVID-19 positive patient might show cavitation in the lung and pleural and pericardial effusion as the disease progresses. Our patient's CT revealed large cavitary lesions in the base of right lung with pleural effusion, which is considered to be a rare manifestation of COVID-19 pneumonia. ${ }^{6}$ In addition, 
chest CT revealed pulmonary nodular lesions and confirmation of Candida species by isolation of the organism by BAL, followed by histopathology, which indicated Candida pneumonia with lung abscess in the patient. ${ }^{17}$ A scoring system based on CT chest findings, termed CORADS, classifies findings into levels of suspicion for COVID-19. The level of suspicion ranges from very low (CO-RADS category 1 ) to very high suspicion (CO-RADS category 5). ${ }^{18}$ Features of CO-RADS category 5 were present in our patient. Thus, prompt recognition of COVID-19 by the CO-RADS category was invaluable to ensure timely treatment and patient isolation.

The ideal treatment of COVID-19 pneumonia has not been clearly determined. Remdesivir, an antiviral drug (suppresses a wide variety of RNA viruses), has shown some clinical benefits (such as lower hospital stays, reduced use of hospital resources and decreased progression to mechanical ventilation) against critical COVID-19 patients. ${ }^{19}$ An August 2020 network meta-analysis of four randomized controlled trials demonstrated that the rate of clinical improvement was significantly higher in the 5-day and the 10-day remdesivir treated groups compared to the standard care group. ${ }^{20}$ Confirmation of the efficacy of remdesivir in hospitalized adult patients with mildmoderate and severe COVID-19 came from the followup of the Adaptive COVID-19 Treatment Trial (ACTT-1) results published in October 2020. ${ }^{21}$ A study from National Institute of Allergy and Infectious DiseasesNational Institute of Health $(\mathrm{NIH})$, USA, reported that patients with severe COVID-19 during hospitalization when treated with remdesivir had a shorter recovery time compared to the placebo and also maximum benefit was observed when remdesivir was given early in the illness. ${ }^{21}$ However, there are contradictory reports about the benefits of remdesivir in COVID-19 patients. $^{19}$ According to a meta-analysis, a substantial number of patients (20$30 \%$ ) has been reported to exhibit serious adverse events or Grade 3 or 4 adverse events during the course of the treatment with remdesivir. ${ }^{22}$ Another systematic review and meta-analysis indicated that remdesivir showed neither benefit against all-cause mortality nor significant treatment efficacy in COVID-19 patients in comparison to placebo/ no treatment groups. Rather patients treated with remdesivir revealed increased risk of nausea than no treatment/placebo groups. Therefore, it should be used only as an experimental drug in patients with severe COVID-19 until sufficient stronger evidence is established based on the findings of randomized controlled studies. ${ }^{23}$ The Food and Drug Administration (FDA) has approved the use of remdesivir for emergency use only in severe/critically ill patients with COVID-19 and the treatment decision is based on the clinical status of the patient and the clinical judgment of the healthcare team. ${ }^{24}$ Hence, remdesivir was started in our patient (based on chest CT findings and severity of illness), while the rtPCR report was awaited.

Our patient was confirmed with a recent right-sided basal ganglia infarct which seemed to be 48 to 72 hours old, indicative of a hypercoagulable state, which is known to be associated with COVID-19. A systematic review and meta-analysis of 27 studies on arterial thrombotic (AT) events in 90 COVID-19 patients reported that approximately $4 \%$ of critically ill COVID-19 patients developed AT events. ${ }^{25}$ Similarly another case series also found hypercoagulability to be correlated with COVID-19. ${ }^{26}$ Multiple arterial sites, including those of the limbs, brain, great vessels, heart, and superior mesentery can get affected in COVID-19. In our patient, results of laboratory investigations corroborated these findings with increased CRP levels and prolonged prothrombin time and INR. Within 24 hours of anticoagulant treatment, our patient also manifested left iliofemoral DVT. Both arterial and venous thromboembolic disease can occur, especially in severe/critically ill COVID-19 patients admitted to the ICU. A multicenter systematic review and meta-analysis among such patients $(\mathrm{N}=9249)$ from China, the USA, UK, and participating countries in Europe revealed that 7.2 to $40.8 \%$ of patients developed thromboembolic events (DVT rather than PE) at some point despite anticoagulation therapy. ${ }^{27}$ Thus, it was suggested that aggressive monitoring of markers of thromboembolic complications such as D-dimer during admission and thromboprophylaxis might be required in managing patients with severe COVID-19 after assessing bleeding risks in each case. A Phase 2 clinical trial (HESACOVID) evaluated whether therapeutic anticoagulation could improve gas exchange compared to the standard anticoagulant thromboprophylaxis, reducing the need to maintain mechanical ventilation in severely ill COVID-19 patients. $^{28}$ Results demonstrated that the patients in the therapeutic enoxaparin group (1 $\mathrm{mg} / \mathrm{kg}$ BID) showed a higher ratio of freedom from mechanical ventilation compared to the prophylactic anticoagulation group. Furthermore, the results of the report also indicated a statistically significant reduction in D-dimer levels over time in the therapeutic enoxaparin group without any major bleeding events. ${ }^{28}$ Likewise, our patient also received enoxaparin and showed 
remarkable improvement in hemodynamic and ventilatory status progressively.

Our patient's status was further complicated when a very high level of HbAlc was detected along with clinical suspicion for oral candidiasis. Increased blood glucose is a risk factor for COVID-19 and should be routinely monitored to improve prognosis in these patients. ${ }^{29,30}$ Moreover, altered cell-mediated immunity and reduced natural killer cell activity affects host response in patients with $\mathrm{DM}$. This can make the patient prone to secondary fungal infections. ${ }^{31}$ High levels of salivary glucose in diabetic patients make them susceptible to oral candidiasis, likely to be secondary to C. albicans infection. ${ }^{32}$ Invasion of the internal organs by commensal Candida facilitates entry of the candida into the blood stream and spread to other sites in the body causing invasive candidiasis. ${ }^{31}$

Laboratory investigations in our patient confirmed the evidence of sepsis (metabolic acidosis, high levels of lactate, and abnormal coagulation variables) with acute kidney injury. Although severe COVID-19 is associated with cytokine storm, no evidence of cytokine storm was found in our patient, thus no anti-inflammatory therapy was considered. In patients with a proven or high likelihood of COVID-19 while awaiting diagnostic test results, evidence-based clinical guidelines recommendations include starting empirical antibacterial therapy to prevent rapid deterioration, if bacterial co-infection was present. ${ }^{33}$ Current estimate points to a risk of up to $20 \%$ secondary infections in severely ill COVID-19 patients. Hence, antibiotic therapy was started, while rtPCR report was awaited. To prevent possible candidemia from the patient's oral mucositis, anidulafungin, which has demonstrated in vitro clinical activity against many Candida species with a high level of efficacy and safety in many multicenter clinical trials, was chosen as the antifungal agent in this case. ${ }^{34}$

It is not uncommon for severely ill patients, especially those who are mechanically ventilated, to become susceptible to HAP. ${ }^{17}$ Since our patient's condition worsened after considerable improvement, he had to be mechanically invasively ventilated when HAP was established. Fourteen days after stabilizing, sudden deterioration in the patient's parameters prompted the clinical suspicion for PE and therefore, CT of chest was performed. Although chest CT did not reveal any evidence for PE, right-sided pleural effusion with large cavitary lesions consistent with lung abscesses was noted. The drained fluid, though bacteria-free, was positive for $C$. albicans, and the antifungal agent was continued till his condition was eventually stabilized, was extubated, and switched to oral medications. The development of Candida necrotizing pneumonia and lung abscess is extremely rare and may be seen in severely immunocompromised patients admitted in the ICU. ${ }^{35,36}$ Prolonged neutropenia, use of broad-spectrum antibiotic therapy, presence of intravascular catheters (with total parenteral nutrition), and intravenous drug use might have been the known predisposing risk factors in our case. Our patient eventually recovered, showed remarkable improvement of health condition and was discharged from the hospital on 14.09.2020 (2 months after emergency admission).

\section{Conclusion}

The unique complexities in this case report highlight the importance of clinical judgment in initiating remdesivir therapy as early as possible in a case suspected of being severely ill with COVID-19. In this case, uncontrolled hyperglycemia, thromboembolic events, and oral candidiasis led to the uncommon manifestation of necrotizing Candida pneumonia with lung abscess. Close monitoring of the patient with timely supportive investigations and therapeutic interventions from an interdisciplinary team aided in the eventual recovery of this patient.

\section{Ethics and Consent Statements}

Written informed consent was obtained from patient's guardian for anonymized patient information to be published in this case report. Approval from the institution was not required to publish the case details in this case report.

\section{Acknowledgments}

Writing assistance was provided by Turacoz Healthcare Solutions (www.turacoz.com). Gilead Sciences provided support to this case report by paying Turacoz Healthcare Solutions for medical writing and managing publication in a peer-reviewed journal.

\section{Disclosure}

The authors report no conflicts of interest in this work.

\section{References}

1. World Health Organization. Pneumonia of unknown cause - China. Disease outbreak news; 2020. Available from: https://www.who.int/ emergencies/disease-outbreak-news/item/2020-DON229. Accessed September 16, 2021. 
2. World Health Organization. Novel coronavirus - china. Disease outbreak news. Available from: https:/www.who.int/emergencies/dis ease-outbreak-news/item/2020-DON233. Accessed September 16, 2021.

3. World Health Organization. WHO director general remarks. Available from: https://www.who.int/director-general/speeches/ detail/who-director-general-s-opening-remarks-at-the-media-briefingon-covid-19-11-march-2020. Accessed September 16, 2021.

4. Aiyegbusi OL, Hughes SE, Turner G, et al. Symptoms, complications and management of long COVID: a review. $J R$ Soc Med. 2021;114:428-442.

5. Xie P, Ma W, Tang H, et al. Severe COVID-19: a review of recent progress with a look toward the future. Front Public Health. 2020;8:189. doi:10.3389/fpubh.2020.00189

6. Caruso D, Polidori T, Guido G, et al. Typical and atypical COVID-19 computed tomography findings. World J Clin Cases. 2020;8:3177. doi:10.12998/wjcc.v8.i15.3177

7. Lai CC, Yu W-L. COVID-19 associated with pulmonary aspergillosis: a literature review. J Microbiol Immunol Infect. 2021;54:46-53. doi:10.1016/j.jmii.2020.09.004

8. Hanson BM, Dinh AQ, Tran TT, et al. Candida auris Invasive Infections during a COVID-19 case surge. Antimicrob Agents Chemother. 2021;65:e0114621. doi:10.1128/aac.01146-21

9. Arastehfar A, Carvalho A, Nguyen MH, et al. Covid-19-associated candidiasis (CAC): an underestimated complication in the absence of immunological predispositions? J Fungi. 2020;6:211. doi:10.3390/ jof6040211

10. Drissi C. Black fungus, the darker side of COVID-19. J Neuroradiol. 2021;48:317-318. doi:10.1016/j.neurad.2021.07.003

11. Singh AK, Singh R, Joshi SR, et al. Mucormycosis in COVID-19: a systematic review of cases reported worldwide and in India. Diabetes Metab Syndr Clin Res Rev. 2021;15:102146. doi:10.1016/ j.dsx.2021.05.019

12. Guan WJ, Ni ZY, Hu Y, et al. Clinical characteristics of coronavirus disease 2019 in China. $N$ Engl J Med. 2020;382:1708-1720. doi:10.1056/NEJMoa2002032

13. Li X, Wang L, Yan S, et al. Clinical characteristics of 25 death cases with COVID-19: a retrospective review of medical records in a single medical center, Wuhan, China. Int J Infect Dis. 2020;94:128-132. doi:10.1016/j.ijid.2020.03.053

14. Ali N. Elevated level of C-reactive protein may be an early marker to predict risk for severity of COVID-19. J Med Virol. 2020;92 (11):2409-2411. doi:10.1002/jmv.26097

15. Smilowitz NR, Kunichoff D, Garshick M, et al. C-reactive protein and clinical outcomes in patients with COVID-19. Eur Heart J. 2021;42:2270-2279. doi:10.1093/eurheartj/ehaa1103

16. Lau AC-W, Yam LY-C, So LK-Y. Management of critically ill patients with severe acute respiratory syndrome (SARS). Int $J$ Med Sci. 2004;1:1. doi:10.7150/ijms.1.1

17. Pappas PG, Kauffman CA, Andes DR, et al. Clinical practice guideline for the management of candidiasis: 2016 update by the Infectious Diseases Society of America. Clin Infect Dis. 2016;62:e1-e50. doi:10.1093/cid/civ933

18. Prokop M, Van Everdingen W, van Rees Vellinga T, et al. CO-RADS: a categorical CT assessment scheme for patients suspected of having COVID-19-definition and evaluation. Radiology. 2020;296:E97E104. doi:10.1148/radiol.2020201473

19. Beigel JH. What is the role of remdesivir in patients with COVID-19? Curr Opin Crit Care. 2021;27:487. doi:10.1097/ MCC.0000000000000866
20. Yokoyama Y, Briasoulis A, Takagi H, et al. Effect of remdesivir on patients with COVID-19: a network meta-analysis of randomized control trials. Virus Res. 2020;288:198137. doi:10.1016/j. virusres.2020.198137

21. Beigel J, Tomashek K, Dodd L. Remdesivir for the treatment of Covid-19-final report. N Engl J Med. 2020;383:1813-1826.

22. Tao J, Aristotelidis R, Zanowick-Marr A, et al. Evaluation of the treatment efficacy and safety of remdesivir for COVID-19: a meta-analysis. SN Compr Clin Med. 2021:1-12. doi:10.1007/ s42399-021-01014-y

23. Okoli GN, Rabbani R, Copstein L, et al. Remdesivir for coronavirus disease 2019 (COVID-19): a systematic review with meta-analysis and trial sequential analysis of randomized controlled trials. Infect Dis. 2021;53:691-699.

24. Health NIo. Antiviral drugs that are approved or under evaluation for the treatment of COVID-19. Available from: https://files.covid19treat mentguidelines.nih.gov/guidelines/section/section_64.pdf. Accessed September 16, 2021.

25. Cheruiyot I, Kipkorir V, Ngure B, et al. Arterial thrombosis in coronavirus disease 2019 patients: a rapid systematic review. Ann Vasc Surg. 2021;70:273-281. doi:10.1016/j.avsg.2020.08.087

26. Mullaguri N, Hepburn M, Gebel JJM, et al. COVID-19 disease and hypercoagulability leading to acute ischemic stroke. Neurohospitalist. 2021;11:131-136. doi:10.1177/1941874420960324

27. Kunutsor SK, Laukkanen JA. Incidence of venous and arterial thromboembolic complications in COVID-19: a systematic review and meta-analysis. Thromb Res. 2020;196:27-30. doi:10.1016/j. thromres.2020.08.022

28. Lemos ACB, Do Espírito Santo DA, Salvetti MC, et al. Therapeutic versus prophylactic anticoagulation for severe COVID-19: a randomized Phase II clinical trial (HESACOVID). Thromb Res. 2020;196:359-366. doi:10.1016/j.thromres.2020.09.026

29. Chen J, Wu C, Wang X, et al. The impact of COVID-19 on blood glucose: a systematic review and meta-analysis. Front Endocrinol. 2020;11. doi:10.3389/fendo.2020.574541

30. Abdi A, Jalilian M, Sarbarzeh PA, et al. Diabetes and COVID-19: a systematic review on the current evidences. Diabetes Res Clin Pract. 2020;166:108347. doi:10.1016/j.diabres.2020.108347

31. Kaur H, Singh D, Rupali KN. Invasive Candidiasis with cavitary lung lesion in a post-Covid-19 diabetic patient-a case report. J Clin Med Res. 2021;3:1-6.

32. Mohammadi F, Javaheri MR, Nekoeian S, et al. Identification of Candida species in the oral cavity of diabetic patients. Curr Med Mycol. 2016;2:1. doi:10.18869/acadpub.cmm.2.2.4

33. Sieswerda E, De Boer MG, Bonten MM, et al. Recommendations for antibacterial therapy in adults with COVID-19-an evidence based guideline. Clin Microbiol Infect. 2021;27:61-66. doi:10.1016/j. cmi.2020.09.041

34. Davis SL, Vazquez JA. Anidulafungin: an evidence-based review of its use in invasive fungal infections. Core Evid. 2007;2:241.

35. Schnabel RM, Linssen CF, Guion N, et al. Candida pneumonia in intensive care unit? Open Forum Infect Dis. 2014;1:ofu026. doi:10.1093/ofid/ofu026

36. Bachh AA, Haq I, Gupta R, et al. Pulmonary candidiasis presenting as mycetoma. Lung India. 2008;25:165. doi:10.4103/09702113.45285 


\section{Publish your work in this journal}

The International Medical Case Reports Journal is an international, peer-reviewed open-access journal publishing original case reports from all medical specialties. Previously unpublished medical posters are also accepted relating to any area of clinical or preclinical science. Submissions should not normally exceed 2,000 words or 4

published pages including figures, diagrams and references. The manuscript management system is completely online and includes a very quick and fair peer-review system, which is all easy to use. Visit http://www.dovepress.com/testimonials.php to read real quotes from published authors.

Submit your manuscript here: https://www.dovepress.com/international-medical-case-reports-journal-journal 